\title{
Design of an environment controlled dew tracking setup to emphasize the role of the relative humidity on breath figures dynamics
}

\author{
Nicolas Pionnier ${ }^{* *}$ (D), Solmaz Boroomandi Barati ${ }^{3}$, Elise Contraires ${ }^{1}$, Rémi Berger ${ }^{2}$, Matthieu Guibert ${ }^{1}$, \\ Stéphane Benayoun ${ }^{1}$ and Stéphane Valette ${ }^{1 *}$
}

\author{
*Correspondence: \\ nicolas.pionnier@doctorant.ec-lyon.fr \\ stephane.valette@ec-lyon.fr \\ ${ }^{1}$ Université de Lyon, Ecole Centrale \\ de Lyon, Laboratoire de Tribologie \\ et Dynamique des Systèmes, UMR \\ 5513, F-69131, 36 Avenue Guy de \\ Collongue, Ecully, France \\ Full list of author information is \\ available at the end of the article
}

\begin{abstract}
In polymer protection pieces, polycarbonate is often used for its transparency and its high impact resistance. However, when dew appears it degrades light transmission and thus transparency. To solve this problem, anti-fogging surfaces are developed. To further understand the dew formation in natural environment in order to design high efficiency anti-fogging surfaces on polycarbonate, a recently designed dew tracking setup giving dew pictures and a digital imaging procedure allowing reliable and highly accurate droplet measurements were developed. Thanks to this setup, dew formation conditions such as relative humidity level and air temperature can be chosen and studied. Particular attention is paid to the evolution of the number of droplets according to the relative humidity and how it explains the droplet growth rate. The influence of the relative humidity $(\mathrm{RH})$ on the growth rate of non-coalescing dropwise condensation has already been observed. This paper is based on these existing results as a comparison to validate the experimental setup and the post-processing analysis. We shall also use the number of droplets to further explain the role of the $\mathrm{RH}$ on condensation dynamics

Moreover, by applying a light subcooling, the growth kinetics were slowed down in order to observe every characteristic phase in detail and even particular intermediate phases.
\end{abstract}

Keywords: Dropwise condensation, Wettability, Relative humidity, Polycarbonate

\section{Introduction}

Dew is the condensation on cold surfaces of water vapor present in a humid environment. Some authors have suggested the use of this phenomenon for water harvesting, improving power plant vapor condensation efficiency or other applied systems [1-5]. These solutions were designed for getting high heat transfer fluxes, increasing the liquid water condensation rate.

In other industrial applications, dew formation is problematic. It can impair surfaces' transparency [6-8] creating security or aesthetic issues because liquid water droplets are assimilated as lenses diffracting light. Droplets can modify light beam direction and generate lighting efficiency losses, furthermore visible droplets can be perceived as a sign of poor quality. To limit the presence of droplets, anti-fogging surfaces are designed by

(c) The Author(s). 2018 licensee Springer on behalf of EPJ. This is an Open Access article distributed under the terms of the Creative Commons Attribution License (http://creativecommons.org/licenses/by/4.0), which permits unrestricted use, distribution, and reproduction in any medium, provided the original work is properly credited. 
modifying the wettability properties $[7,9,10]$. These surfaces are created by managing chemical and/or topographical properties. Two approaches exist - reducing the presence of water on surfaces (hydrophobic surface treatments) or spreading droplets to create a uniform water film (hydrophilic surface treatments) [11, 12].

In industry, two types of transparent materials are frequently used - polymers and glass. Among these two families, three materials are the most commonly used - polycarbonate, polymethyl methacrylate (PMMA) and glass. The latter two have the best transparency properties [13]. However, polycarbonate has the best impact resistance (250 times higher than ordinary glass and 40 times higher than PMMA) and has a good thermic resistance [14]. Thanks to its properties, polycarbonate is used for transparent protection pieces such as plane windows, front car headlights, motorcycle helmet visors or diving masks.

All these cited industrial applications undergo different climatic conditions in their typical uses such as different subcooling temperatures, air temperatures or levels of relative humidity (RH). Modifying these parameters can change the droplet density of a breath figure (condensed droplet pattern) and with it the dynamics of dropwise water vapor condensation.

By tracking droplets and their radius $\mathrm{R}$, the kinetics of condensation can be described. This kind of study is based on monitoring the number of droplets and the droplets radii as a function of time. The droplets radii as a function of time are also called growth rate. Two distinct cases can be highlighted - the isolated droplet $[15,16]$ with a radius which increases because of the direct incorporation of water vapor and follows $R \sim t^{1 / 2}$ and the densely packed array of droplets where droplets have to share the water vapor. In the latter case, droplets are closed and their neighboring water vapor concentration profiles overlap thereby decreasing the water supply of each one. In this configuration, droplets nucleation and growth can be divided into three phases (illustrated in Fig. 1 taken from Macner et al. work [2]) [2, 17].

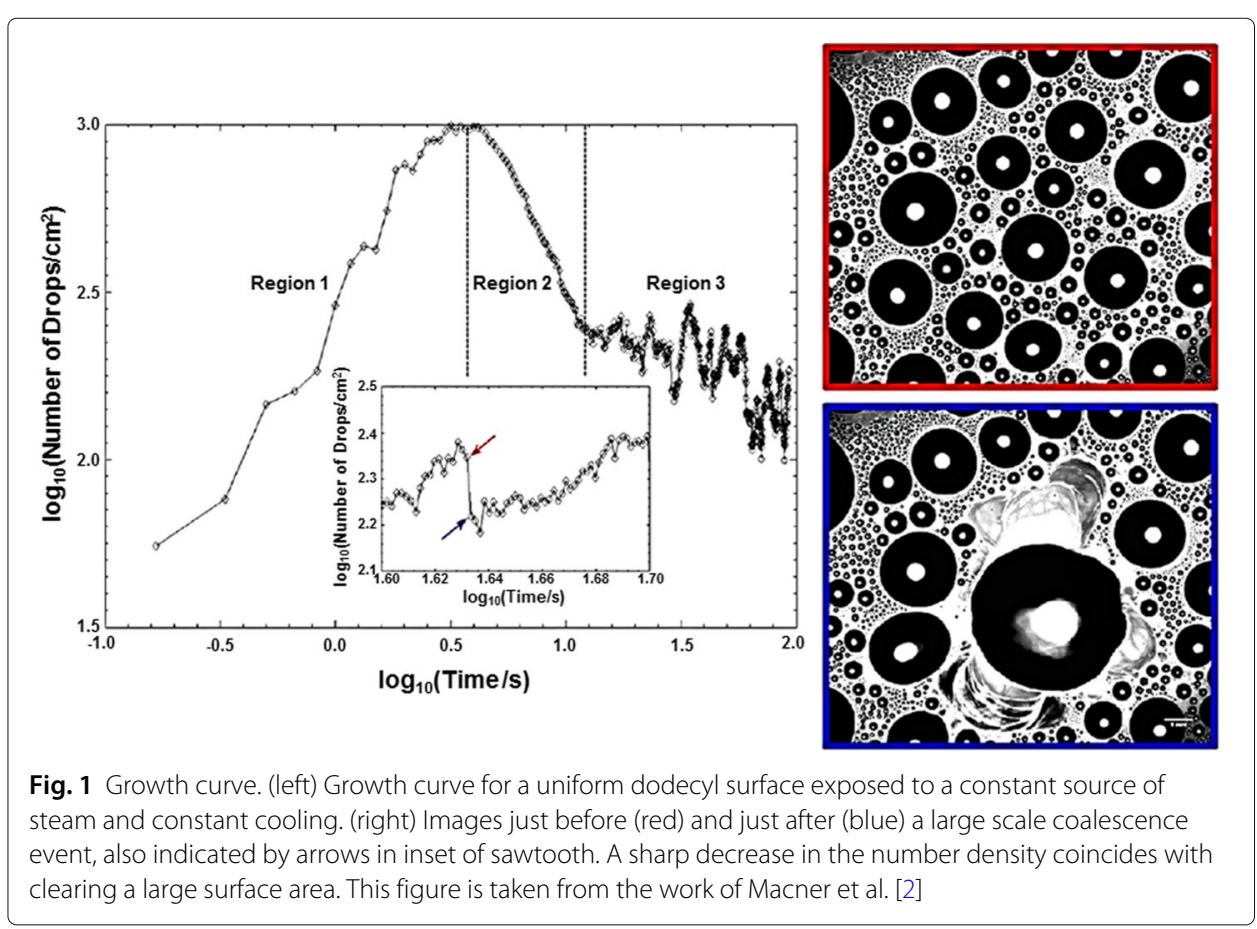


The first phase (region 1 in Fig. 1) is the formation of liquid droplets characterized by small droplets growing only through the direct incorporation of water vapor. This mechanism is defined by a mean droplet radius growth of $<R>\sim t^{1 / 3}$. The $1 / 3$ exponent depends on the relative humidity of the air and increases with it [18]. When growing, droplets touch each other and coalesce. This is the second phase (region 2 in Fig. 1). The number of droplets decreases quickly and the droplets grow following $<R>\sim t$. The last phase (region 3 in Fig. 1), observed when surfaces are cooled, begins once droplets are sufficiently large [2]. The coalescence now creates free spaces where new droplets nucleate. The balance between the older bigger droplets and the new little ones induces the stagnation of $\langle R>$ and the number of droplets. $\langle R>$ is nevertheless characterized by quick variations corresponding to the different coalescence events.

Castillo et al. [18] showed that relative humidity has an impact on dew formation. On coated smooth silicon samples, they demonstrated that the growth rate of non-coalescing droplets increases with $\mathrm{RH}$. Thus, they fixed the sample surface subcooling temperature at $15{ }^{\circ} \mathrm{C}$, maintained the air temperature at $20{ }^{\circ} \mathrm{C}$ at the ambient pressure and varied the RH to $45 \%, 50 \%, 55 \%$ and $70 \%$.

To improve anti-fogging treatments, this study reproduced dew formation under different relative humidity levels. In this purpose, this paper shows a custom-made environment controlled dew tracking apparatus giving dew image sequences combined with a digital imaging procedure. This method allowed accurate wide size range dew droplets tracking for large studied areas.

Both the designed setup and the post-processing analysis were validate thanks to the work of Castillo et al [18]. Moreover, beyond their work, this paper also explains the variation of the dynamics of condensation growth with respect to relative humidity through droplet density (the number of droplets). The time condensation dynamics were studied at low relative humidity levels $(40 \%, 45 \%$ and $60 \%)$ and low subcooling levels to obtain low condensation kinetics in order to observe potential intermediate phases. The subcooling was set at $1^{\circ} \mathrm{C}$ and the pressure was at the ambient level.

\section{Experimental setup and methods}

\section{Condensation test apparatus and data post-processing}

Description of the condensation test apparatus

A specific custom-made setup was developed for the experiment. This apparatus produced and allowed observation of condensation on a sample surface by decreasing its surface temperature below the dew point temperature in an atmosphere controlled in temperature and relative humidity $(\mathrm{RH})$.

A schematic drawing of the experimental setup is shown in Fig. 2. The humid air was generated in a flow circuit where room air was mixed with water-saturated air. The room air was compressed and filtered with a $0.01 \mu \mathrm{m}$ gas filter. Because of the compression and depending on the relative humidity of the room, the $\mathrm{RH}$ of the air decreased to below $20 \%$, which was low enough for the required test conditions. This 'dry' compressed air could then be redirected into a gas humidifier to obtain water-saturated air. The saturated air was mixed thanks to a proportional valve with compressed room air (under $20 \% \mathrm{RH}$ ) to obtain the desired RH. Afterwards, the humid air at the required level was piped to the test chamber where a hygrometer (HMP 110, Vaisala) continuously measured the relative humidity and provided feedback to a PID control loop (Eurotherm 3216) controlling the 


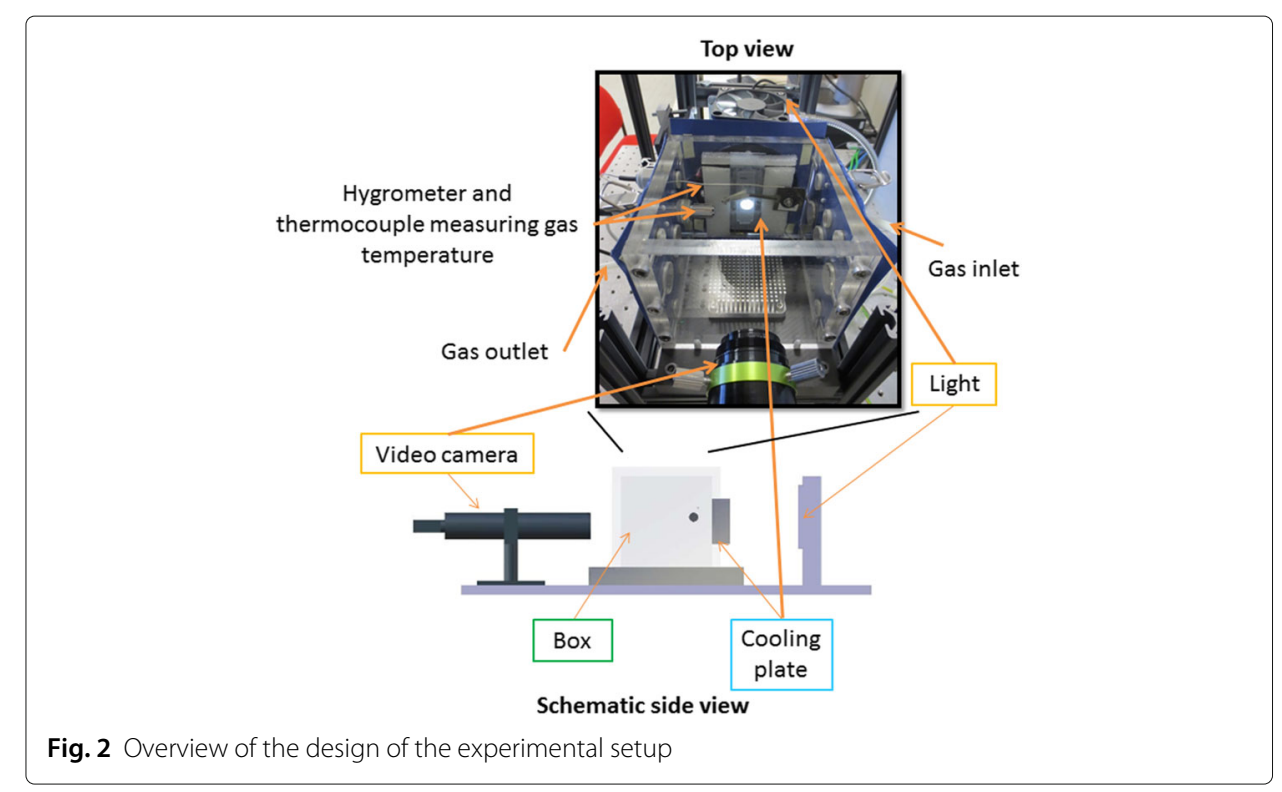

valve and thus the relative humidity in the chamber. The gas humidifier was heated to balance the fall in temperature during vaporization.

The pressure and flow rate were respectively set at 2 bars and to $1.15 \mathrm{~L} / \mathrm{min}$ at the output of the compressor (before the proportional valve) to protect the different components. The flow rate was also measured just ahead of the chamber to obtain an exact reading of the input in the chamber.

The test chamber was a $10 \times 10 \times 10 \mathrm{~cm}^{3}$ transparent polycarbonate box with an aluminum soleplate and equipped with a gas inlet and an outlet evacuating gas to the room. The pressure inside the chamber was thus equal to the room pressure considered as constant during condensation experiments. In this chamber, the relative humidity, the gas temperature and the temperature of the sample surface were controlled. Two 150-watt heating cartridges were placed inside the aluminum soleplate in contact with a thermal diffuser which heated the chamber air by convection. The sample was fixed with thermal paste (MX-4, Arctic) to an aluminum plate also fixed to two 23.4 Watts thermoelectric coolers (UEPT-1RE-063-050M125S, UWE Electronic). The hot side of the thermoelectric coolers was in contact with a thermal diffuser with two fans outside the chamber. Therefore, the cooling of this side depended on the room temperature.

Thanks to two $1 \mathrm{~mm}$-diameter K-type thermocouples and two other Eurotherm 3216 controlling the cartridges and the thermoelectric coolers, the two related temperatures could be set as constant. The gas temperature was measured at $20 \mathrm{~mm}$ of the aluminum plate and the subcooling temperature was measured in the cold aluminum plate. Depending on the sample, the surface sample temperature could vary from the measured temperature. Each sample surface temperature was thus measured and compared to the given subcooling temperature.

The sample surface observations were performed at normal incidence through a polycarbonate wall of the chamber with a $5 \mathrm{Mpx}$ grayscale video camera (MANTA G-504B, Allied Vision) mounted on a long-focal-distance adjustable lens (QIOPTIQ). This system allows a spatial resolution ranging from $0.28 \pm 0.03 \mu \mathrm{m} /$ pixel to $2.60 \pm 0.08 \mu \mathrm{m} /$ pixel. 
The video camera was mounted outside the box to avoid contact with the high humidity atmosphere. A through hole was drilled into the cooling sample assembly to allow sample backlight. The light was a $97.04 \%$ uniform $50 \times 50 \mathrm{~mm}^{2}$ white screen (SLLUB, PHLOX) placed outside the chamber in front of the hole. As shown in Fig. 3a and b, droplets were seen as dark on the contrasting light background. For a better alignment between the video camera, the sample and the light and also to avoid vibrations, all the visualization components and the test chamber were fixed to an optical table (Vision IsoStation, Newport). Moreover, to simplify focusing and facilitate more precise displacements on the sample surface, the video camera and the adjustable lens were mounted on a XYZ micrometer stage.

To remove contrast variation because of external light evolution (like natural sun light variation along the day) on the images obtained, all the apparatus was enclosed in an aluminum plate structure.

The experimental data (temperatures, relative humidity and condensation images) were recorded at regular time intervals with a homemade LabVIEW program.

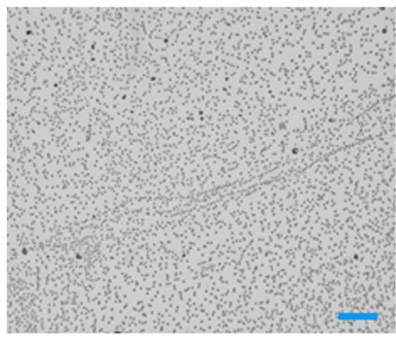

a

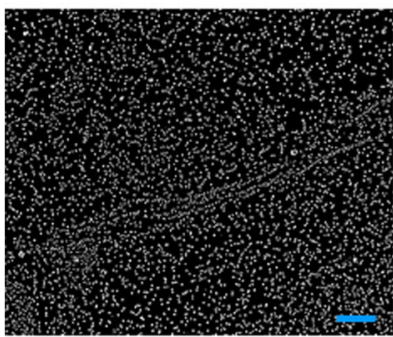

C

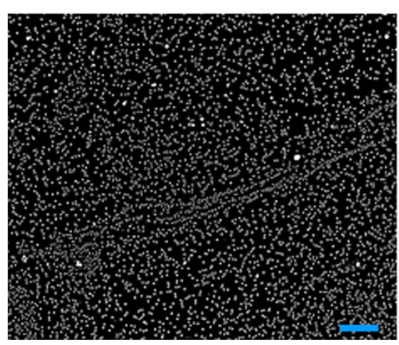

e

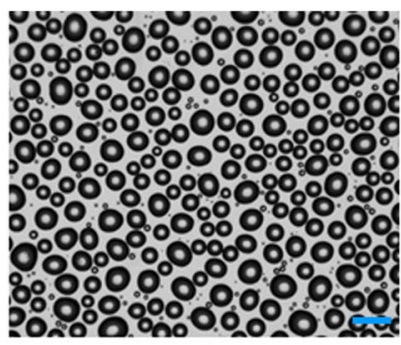

b

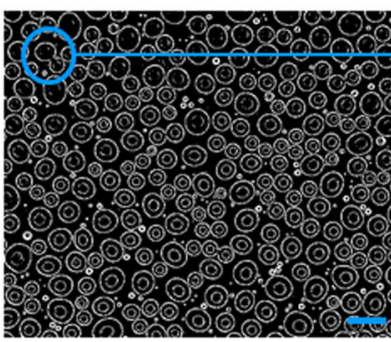

d

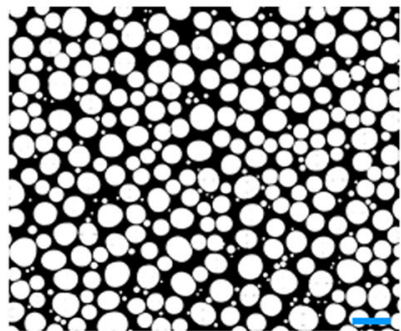

f

Fig. 3 Exemple pictures of a condensation test illustrating the use of the two droplets detection programs. Exemple pictures of a $45 \%$ relative humidity level, $30^{\circ} \mathrm{C}$ air temperature and $1{ }^{\circ} \mathrm{C}$ subcooling condensation test illustrating the use of the two droplets detection programs. $\mathbf{a}, \mathbf{c}, \mathbf{e}$ are respectively the raw images, the enhanced droplet profiles and the final binary images obtained with the first program. $\mathbf{b}, \mathbf{d}, \mathbf{f}$ are respectively the raw images, the enhanced droplet profiles and the final binary images obtained by the second program. The scale bar represents $320 \mu \mathrm{m}$ 


\section{Data post-processing}

Raw images (Fig. 3a and b) obtained with the use of the apparatus needed to be posttreated to detect droplets and characterize their position, area and radius. Two MATLAB scripts, based on the same image treatment, were developed - one for small droplets present at the very beginning of condensation (program 1) and another for bigger droplets (program 2). At the beginning (program 1), detection errors were rare avoiding complex treatments necessary in program 2 . The two programs pretreated images by applying the discrete Laplace operator to reduce contrasts inhomogeneity by accentuating dark/bright interfaces, thus giving droplet profiles [19] (see Fig. 3c and d). The enhanced images were segmented to binary images using Otsu's method - droplet profiles appeared in white on a black background [20]. In order to close profiles and obtain droplets, a close operator was used and the image edges were manually turned white, reducing the studied area but closing the partially-seen droplet profiles. Then, all black pixels not belonging to the background and circle by white pixels were turned in white to fill all droplet profiles. However, some droplets were connected in clusters and a watershed function was used to dissociate them.

After these operations, not all the droplets could be filled because some opened profiles remained. This kind of error occurred mainly when the obtained profiles were not continuous (see the magnified picture of Fig. 3d). This happened for high droplet perimeter (for droplets with diameters larger than $26 \mu \mathrm{m}$ ). To solve these errors mainly present in program 2 (treating large droplets), a new function was added to suppress the remaining droplet profiles and surface defaults. This was not the case with program 1 which thus enabled a lower detection threshold to be attained and explains its use with small droplets at the beginning of condensation. Moreover, during the use of program 1, the number and area of detected surface defaults were negligible.

With both programs, the final undetected droplets were low and smoothed with the high number of taken images. A droplet could be undetected on one image and detected on the next one.

\section{Surface characteristics}

The polycarbonate surfaces were characterized by topographical analysis and by measuring the static, advancing and receding contact angles.

The polycarbonate surfaces were obtained by molding injection of the Makrolon 2458 from Bayer. The polycarbonate samples were replicated from a mirror polish with a low roughness injection mold [21].

The topographical characterization was carried out using atomic force microscopy (NanoscopeV NanoMan Bruker Corporation). The topography of the surfaces was measured on three different scans of $10 \mu \mathrm{m}^{2}$ on an arbitrary place of the surface. The obtained topographies were studied with the DigitalSurf software AltiMap. The mean roughness parameters $S q$ and $S a$ on PC substrate were respectively $S q / \mathrm{nm}=8.9 \pm 3.3$ and $\mathrm{Sa} / \mathrm{nm}=7.0 \pm 2.7$.

For static contact angle experiments, a Kruss DSA30 tensiometer was used to deposit 3 $\mu \mathrm{l}$ sessile drops at different locations of the studied area. For the purposes of a statistical approach, ten drops were deposited.

For the advancing and the receding contact angles, the same method was used as for the static contact angle. However, in this case water was progressively injected into the 
droplet (to obtain the advancing contact angle) and then pumped out of it (to obtain the receding contact angle) [22]. In both cases, when the drop base line started moving, the respective advancing and receding contact angles $\left(\theta_{\mathrm{a}}\right.$ and $\left.\theta_{\mathrm{r}}\right)$ were measured. The difference between these two respective angles is defined as the contact angle hysteresis of the surface $(\Delta \theta)$. For our samples, the results are: $\theta /{ }^{\circ}=87 \pm 1, \theta_{\mathrm{a}} /^{\circ}=93 \pm 3, \theta_{\mathrm{r}} /^{\circ}=34 \pm 6$ and $\Delta \theta /{ }^{\circ}=59 \pm 7$.

\section{Experimental procedure Preparation of surfaces}

According to the supplier, the Makrolon 2458 polycarbonate has a water absorption that can reach $0.30 \%$. If the water absorption rate is not controlled, it will generate variability in the wetting properties. Surface preparation is thereby a critical parameter for good condensation test repeatability.

The samples must be cleaned before condensation tests in order to remove pollution. Indeed, dirt particles can create preferential nucleation sites favoring the formation of water droplets and altering the potential surface condensation properties [23].

The polycarbonate chemical properties must also be preserved. As seen in the polycarbonate chemical compatibility table, polycarbonate has a good resistance to 96\% diluted ethanol (volume concentration) and to water at room temperature [24]. Nevertheless, we chose to use a $70 \%$ diluted ethanol to avoid any harmful chemical interactions. Moreover, polycarbonate was only to be briefly in contact with ethanol.

The cleaning was carried out in four steps:

1 Seventy Percent diluted ethanol was used to remove the grease,

2 the sample was cleaned with distilled water and was placed for $15 \mathrm{~min}$ in a distilled water ultrasound bath to remove dust,

3 the sample was dried with nitrogen to remove liquid water from the surface,

4 the sample was placed in an oven at $120^{\circ} \mathrm{C}$ for six hours to remove any water molecules on the surface and absorbed by the polymer.

\section{Experimental condensation procedure}

Condensation experiments were defined by a specific relative humidity, gas temperature and subcooling temperature. Firstly, the sample was bound and the box lid was closed. Before initializing condensation, the gas humidifier temperature, gas temperature inside the box and relative humidity needed to be stabilized at the desired set points. Two hours were required to reach such stabilized points. Afterwards, thermoelectric coolers were turned on and the desired subcooling temperature was reached in less than 2 min. The condensation starting point parameters were thus well controlled. The gas temperature and relative humidity were constant and the dew point temperature was quickly reached thanks to the fast thermoelectric cooler response. Thanks to that, the measured parameters at the first detected droplets was constant and had already reached their setpoint.

In these experiments, the gas temperature was set at $30^{\circ} \mathrm{C} \pm 2.5^{\circ} \mathrm{C}$, the relative humidity at $40 \% \pm 1.6 \%, 45 \% \pm 1.6 \%$ and $60 \% \pm 1.6 \%$ and the surface subcooling temperature at about $1{ }^{\circ} \mathrm{C}$. 
The room temperature was also set at around $23^{\circ} \mathrm{C}$ to allow a repeatable cooling of the thermoelectric elements. The subcooling temperature was not controlled with the surface sample temperature but instead with the thermocouple located in the cold aluminum plate, so an abacus was developed to find the sample surface temperature at the observation point as a function of the aluminum plate temperature.

The room temperature was set at about $23^{\circ} \mathrm{C}$ and the temperature decreasing range up to the subcooling temperature was set constant to allow a repeatable and identical cooling of the thermoelectric elements between the different tests. The subcooling temperature was not controlled with the surface sample temperature but instead with the thermocouple located in the cold aluminum plate, so abacus were developed to find the sample surface temperature at the observation point as a function of the aluminum plate temperature. For a better accuracy, an abacus for each relative humidity level was needed. Three trials were done to make an abacus. The most representative of these three was kept and the others were used for statistics. The abacus were linear equation $-T_{\mathrm{s}}=\mathrm{A} \cdot \mathrm{T}_{\mathrm{al}}+\mathrm{B}$ with $\mathrm{T}_{\mathrm{s}} /{ }^{\circ} \mathrm{C}$ the surface temperature at the observation point and $\mathrm{T}_{\mathrm{al}} /{ }^{\circ} \mathrm{C}$ the aluminum plate temperature. The A and B coefficients are given in Table 1.

With such low relative humidity levels and subcooling temperatures, the condensation kinetics was relatively slow allowing observation of the droplets appearance and intermediate phases. A test took about four hours.

The experimental data (temperatures, relative humidity and condensation images) were recorded at $1 \mathrm{~Hz}$ acquisition rate. The sequence of images was taken with a 0.75 magnification factor and with set video camera parameters. The spatial image resolution was $1.34 \pm 0.04 \mu \mathrm{m} /$ pixel. The observed area was approximately $3.3 \times 2.7 \mathrm{~mm}^{2}$ and the smallest detected droplet diameter was 5 pixels $(=6.7 \pm 0.2 \mu \mathrm{m})$.

\section{Results and discussion \\ Setup validation tests \\ Breath figure growth}

Figure 4 shows the number of droplets as a function of time for the $45 \%$ relative humidity condition test. The three expected regions $[2,18]$ are well defined and another can also be described - droplet appearance.

[0 s - 22 s] Step 1: Droplet appearance. During this step, droplets which had nucleated and grown by direct incorporation of water vapor and by small length scale coalescence events exceed the experimental droplet radius detection threshold. The appearance was progressive starting from the coldest places (the observation hole borders) and filling the whole studied area when the PC temperature decreased to below the dew point temperature. In this transient step, the number of detected

Table 1 Table of the abacus equations coefficients

\begin{tabular}{lllllll}
\hline $\begin{array}{l}\text { Equation } \\
\text { coefficients }\end{array}$ & $\mathrm{A}(40 \% \mathrm{RH})$ & $\mathrm{B}(40 \% \mathrm{RH})$ & $\mathrm{A}(45 \% \mathrm{RH})$ & $\mathrm{B}(45 \% \mathrm{RH})$ & $\mathrm{A}(60 \% \mathrm{RH})$ & $\mathrm{B}(60 \% \mathrm{RH})$ \\
\hline $\begin{array}{l}\text { Representative } \\
\text { coefficients }\end{array}$ & 0.6783 & 10.202 & 0.6584 & 10.864 & 0.6574 & 10.909 \\
$\begin{array}{l}\text { Mean } \\
\text { coefficients }\end{array}$ & 0.6823 & 10.2573 & 0.6680 & 10.6105 & 0.6654 & 10.6923 \\
\hline
\end{tabular}

The representative coefficients are the coefficients of the chosen representative abacus. The mean coefficients and the corresponding standard deviations are obtained with the three trials 


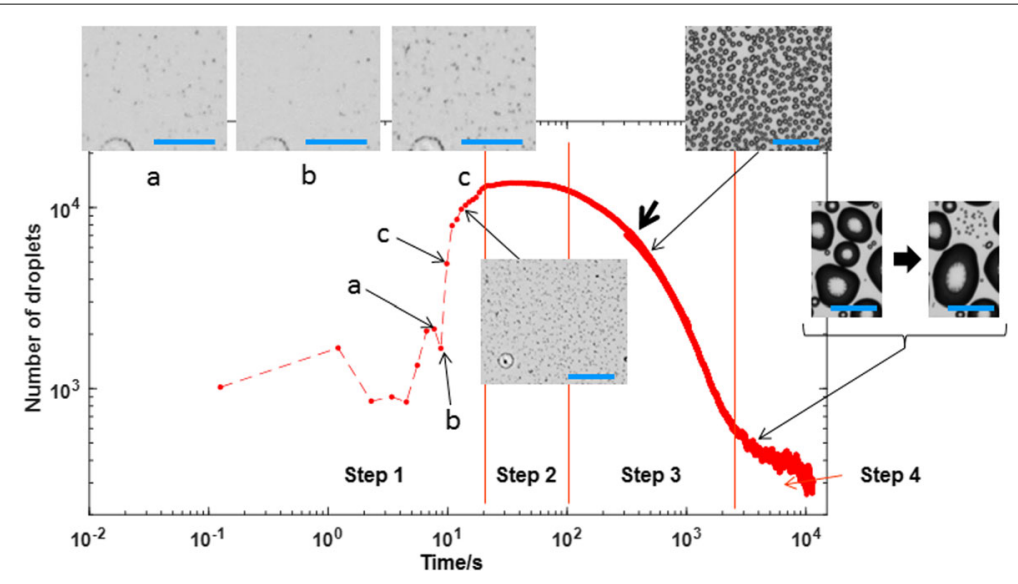

Fig. 4 Number of droplets as a function of time for the $45 \%$ relative humidity. The smallest detected droplet diameter is 5 pixels (droplet diameter $6.7 \mu \mathrm{m}$ ). Pictures are part of raw images. The scale bar represents 160 $\mu \mathrm{m}$. Pictures $\mathbf{a}, \mathbf{b}$ and $\mathbf{c}$ represent evaporation/re-condensation event

droplets increased with the decrease of the surface temperature and some successive evaporation and re-condensation events , probably due to RH fluctuations, were observed (an example is given in Fig. 4, pictures a, b and c). At the end, this region was made up of small droplets with low variation in their size. This step 1 is due to the dew set up temperature becoming lower that of the dew point.

[22 s - 63 s] Step 2: Droplet growth without coalescence. This step is represented by the stagnation of the number of droplets. Droplets were growing because of the addition of water vapor.

[63 s - $43 \mathrm{~min}$ ] Step 3: Droplet growth with coalescence events. It is characterized by a decreasing number of droplets. When droplets are big enough, new droplets appear in the free spaces left by coalescing droplets. As long as coalescence events exceed the number of droplets appearing, there is a decrease in the number of droplets characteristic of the step 3.

[43 min - end] Step 4: Number of droplets plateau. The decreasing number of droplets will reach a plateau because coalescence events and the appearance of droplets in the free spaces counterbalance each other and stabilize the number of droplets. This step continues after data acquisition until a large droplet cleans the entire studied area starting again the whole process to step 1.

The transition between the two data processing programs created an error represented by the bold black arrow on Fig. 4. This was due to the difference in the detection threshold. This error can be seen in each experimental curve. An overlapping of the two programs in this region has been carried out to help readers understand better.

The evolution of the number of droplets shown in Fig. 4 corresponds to the literature (Fig. 1 from Macner et al. [2]) and thus validates our experimental setup and methods. However, in order to ascertain the reliability of our setup, the droplet growth rate was studied.

\section{Droplet growth rate}

Another method to describe breath figures growing is using the growth rate represented by the mean radius of a droplet array as a function of time. The different steps are defined 
by the exponent $\mu$ of the relation $<R>\sim t^{\mu}$. The growth rate of the $45 \%$ relative humidity breath figure, given in Fig. 5, is analyzed.

As explained in literature, the growth rate evolution with time of a breath figure occurs over three distinct phases representing well defined growing mechanisms [17, 25, 26]. The first phase, in which droplets grow by direct incorporation of water vapor, follows $<R>\sim t^{1 / 3}$.

In a steady state, water incorporation of a densely packed array of droplets is considered as constant. By calculating the corresponding vapor flow, the evolution of droplets volume due to absorption is [15]:

$$
\frac{d V}{d t} f_{v} V_{\text {mol }}<d>\Rightarrow V \sim t
$$

with $f_{v}$ the vapor flow, $V_{m o l}$ the molar volume, $<d>$ the mean distance between drop centers and $\mathrm{V}$ the total condensed volume. For a droplet of the pack (i): $V_{i} \sim t$ and $R_{i} \sim$ $t^{1 / 3}$. Thereby, the mean droplet growth is $<R>\sim t^{1 / 3}$.

Afterwards, in the second phase, $\mu$ becomes equal to one with the appearance of coalescence events. When coalescence and new droplets appearance are counterbalanced, the mean droplet radius $<R>$ becomes constant ( $\mu$ tends to 0 ). This is the third phase, also described by a stagnation of the number of droplets (see step 4 in Fig. 4).

Coalescences bring randomness which prevents an easy and precise analytical explanation of the second and third phases exponents. However, the given tendencies were largely approved in literature $[2,15,17,18]$.

Figure 5 shows the three phases predicted by the theory. These are represented as phases I, II and III. The measured exponent $\mu_{I}$, corresponding to the first phase of the theory and to the step 2 in Fig. 4, is $0.29 \pm 0.01$. This value is comparable to the experimental values seen in literature, close to $1 / 3[27,28]$. For the theoretical second phase (phase II, Fig. 5), the measured exponent is $0.81 \pm 0.01$ which is compatible with the reported values between 0.75 and 1.03 [15, 27, 29, 30]. Phase III, the last phase described by theory, $<R>$ is the stagnation. Phase 0 is the droplets' appearance corresponding to the step 1

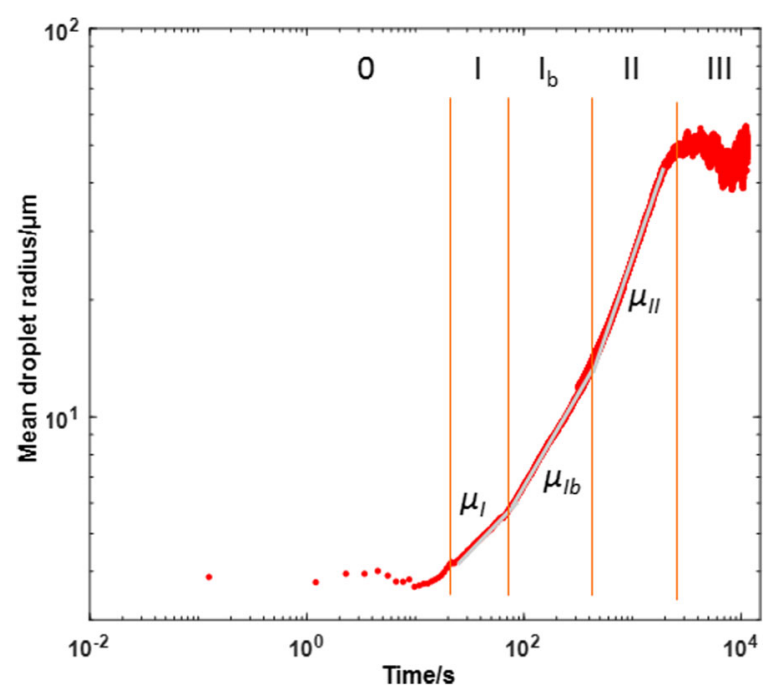

Fig. 5 Mean droplet radius as a function of time for the $45 \%$ relative humidity. $0, I, I_{b}, I I$ and III describe the different phases 
described in Fig. 4 and phase $I_{b}$ is an intermediate phase between non-coalescing phases ( 0 and I) and the coalescing phases (II and III). In this phase, coalescence events begin but are not important enough to obtain a $\mu$ value of about 1 . The measured $\mu$ exponent is $0.48 \pm 0.01$. The exponent $\mu_{I_{b}}$ tends to $\mu_{I I}$ with the increased importance of coalescence events.

To conclude this section, the evolution of the growth factor through the succeeding phases is explained by droplet interactions:

- 0: droplets appearance,

- I: no droplet interaction (theory: $\mu_{I} \rightarrow 1 / 3$; experiment: $\mu_{I}=0.29 \pm 0.01$ )

- $I_{b}$ : beginning of coalescence events (theory: $\mu_{I_{b}}$ is reaching 1 ; experiment: $\left.\mu_{I_{b}}=0.48 \pm 0.01\right)$

- II: large coalescence events (theory: $\mu_{I I} \rightarrow 1$; experiment: $\mu_{I I}=0.81 \pm 0.01$ ),

- III: stabilized phase $\left(\mu_{I I I} \rightarrow 0\right)$.

By comparing our results with the literature, we can certify the reliability of our experimental setup and data processing.

\section{Condensation dynamic evolution with relative humidity}

The breath figure growth and the droplets growth rate were studied for different relative humidity levels (40\%, 45\% and $60 \%$ ).

\section{Breath figure growth}

By varying the relative humidity $(40 \%, 45 \%$ and $60 \%)$ for a constant subcooling temperature of $1^{\circ} \mathrm{C}$, the number of droplets can be modified (Fig. 6). The 4 steps observed were still present and characteristic. The maximum number of droplets was found to decrease with relative humidity from 24450 droplets for $40 \%$ to 4963 droplets for $60 \%$. As seen in Fig. 6, the number of droplets was higher at low relative humidity. Moreover, the maximum number of droplets appeared sooner with relative humidity decreasing the droplet appearance (step 1) duration. According to Castillo et al. [18], the rate of condensation of small droplets increases with relative humidity, thereby droplets grow faster decreasing the duration of droplet appearance (step 1). During this transient step, little droplets can easily evaporate because of any small variation of condensation parameters. This explains the presence of evaporation and re-condensation events (an example is given Fig. 4, pictures a, b and c). These events are at the origin of the increase of the number of droplets. The first detected droplets seem to inhibit the nucleation of new droplets avoiding this increase of the number of droplets. An inhibited region is defined as the place where the supersaturation is not high enough to allow nucleation.

Indeed, nucleation cannot take place if the inhibited region around a droplet is higher than half of the distance between two droplets $[26,31]$. This can be written as:

$$
2 \delta<(<d>)<=>\frac{2 \delta}{<R>}<\frac{<d>}{<R>}<=>2 \frac{p_{\infty}-p_{s}}{p_{\infty}-p_{0}}<\frac{<d>}{<R>}
$$

with $\delta$ the radius of the inhibited region of a droplet and $\langle d>$ the mean distance between drop centers. $p_{\infty}, p_{s}$ and $p_{0}$ represent respectively the saturation pressure of water far from the substrate, the saturation pressure of water on the substrate surface and the saturation pressure of water taking into account the supersaturation. Thanks to these pressures, $\delta$ could be calculated. In the present experimental situation, for $40 \%, 45 \%$ and 


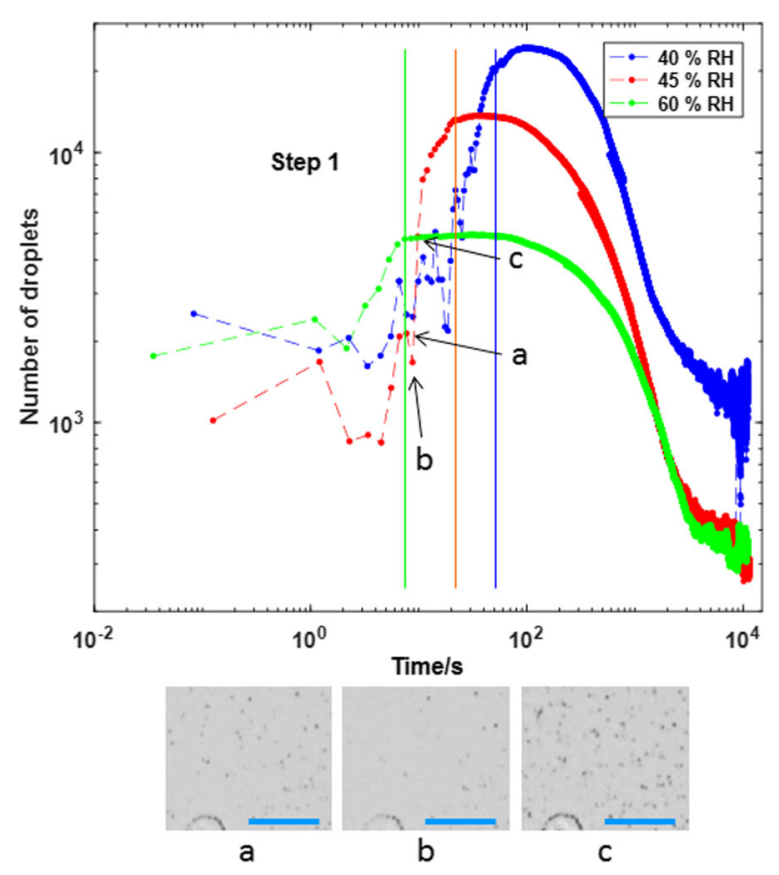

Fig. 6 Number of droplets as a function of time for the $40 \%, 45 \%$ and $60 \%$ relative humidity. Pictures (a), (b) and (c) represent evaporation and re-condensation event during the droplet appearance step for the $45 \%$ relative humidity. The scale bar corresponds to $160 \mu \mathrm{m}$

$60 \%$ relative humidity levels, $\delta /<R>$ was found to be between 1.04 for $40 \% \mathrm{RH}$ and 1.09 for $60 \% \mathrm{RH}$ whereas $<d>/<R>$ obtained during step 1 were between 3.2 for $40 \% \mathrm{RH}$ and 5.2 for $60 \% \mathrm{RH}$.

Consequently, according to the inequation 2, new droplets can nucleate between the existing ones. However, experimentally it did not seem to be the case. No droplet appears in the existing condensation figures. It is possible that nucleated droplets are not detected by the experimental set up. To validate this theory, distances between droplets should be studied.

The mean spacing between each droplet and its closest neighbors $\left\langle d_{b}>\right.$ as a function of time is given in Fig. 7. As seen in Fig. 7, at low relative humidity, droplets are closer to each other and the breath figures are more densely packed. This phenomenon is due to the step 1 duration. The longest the drop appearance, the most numerous evaporation and re-condensation events.

At the end of the step $1,<d_{b}>$ is about $5.3 \mu \mathrm{m}$ for $40 \% \mathrm{RH}, 7.1 \mu \mathrm{m}$ for $45 \% \mathrm{RH}$ and $15.7 \mu \mathrm{m}$ for $60 \% \mathrm{RH}$ (see Fig. 7). For $40 \% \mathrm{RH}$ and $45 \% \mathrm{RH}$, spacing between droplets edge is lower or almost similar to the smallest detectable droplet diameter $(=6.7 \pm 0.2 \mu \mathrm{m})$. Nucleated droplets are thus incorporated before being observed. For $60 \% \mathrm{RH}$, the distance $15.7 \mu \mathrm{m}$ is not high compared to the detection threshold, it is thus possible that droplets are incorporated before their detection. Distance between existing droplets have to increase in order to allow new droplets appearance. This should be the case during evaporation and re-condensation events. After the disappearance of droplets, they systematically appeared again with new droplets between them (as seen in the pictures a, b and c, Figs. 4 and 6). Thereby, by increasing the growth rate with the relative humidity, 


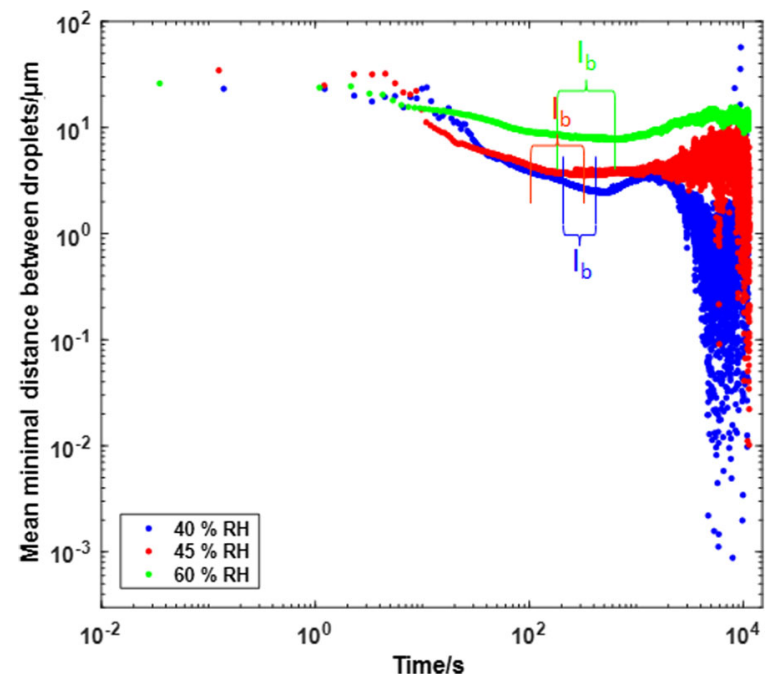

Fig. 7 Mean spacing between each droplet and its closest neighbor $<d_{b}>$ as a function of time. The measured distances are edge to edge. $I_{b}$ describe phase $I_{b}$

droplets were found to be less prone to evaporation because of its larger size and thus new droplets could not appear between the existing ones.

\section{Droplet growth rate}

By studying droplet growth rates with respect to the relative humidity levels, Castillo et al. [18] found that the theoretical exponent $\mu=1 / 3$ depends on the relative humidity. They observed an increase of this exponent with respect to relative humidity. Moreover, regarding their work, the exponent $\mu=1$, which appears when coalescence occurs, found to be not impacted by the relative humidity. Coalescence events overcome the effect of relative humidity. Growth by direct incorporation of water vapor $(\mu=1 / 3)$ varying with $\mathrm{RH}$ is negligible compared to growth with coalescence which does not depend on RH but on droplet size and distance between them.

Figure 8 shows the growth rates obtained from the different sets of relative humidity. To describe these different curves the same nomenclature as in Fig. 5 is used for each phase. The exponent $\mu$ of the different phases are given in Table 2.

As shown by Castillo et al., relative humidity has an influence on phase I [18]. The growth rate $\mu_{I}$ increases with the relative humidity. According to them, this variation is due to the increase of condensation mass flux with respect to the relative humidity for droplets of relatively small radii. Droplets grow faster because of the addition of water supply. However, we found that this water vapor supply also controls the number of droplets appearing thus controlling the amount of vapor shared between them. The number of droplets decreases with the RH. The vapor is thus shared between a reduced number of droplets. The increase in phase $\mathrm{I}_{\mathrm{b}}$ growth rate thus not only depends on direct addition of water supply but also on the decreased number of droplets sharing the water vapor in the air.

In phase $\mathrm{I}_{\mathrm{b}}$, coalescence events start and the number of droplets governs the growth rate. By decreasing the number of droplets, the space between droplets increases which then causes the number of coalescence events to decrease. The decrease in the factor $\mu_{I_{b}}$ with respect to the relative humidity is explained by the distance between droplets 


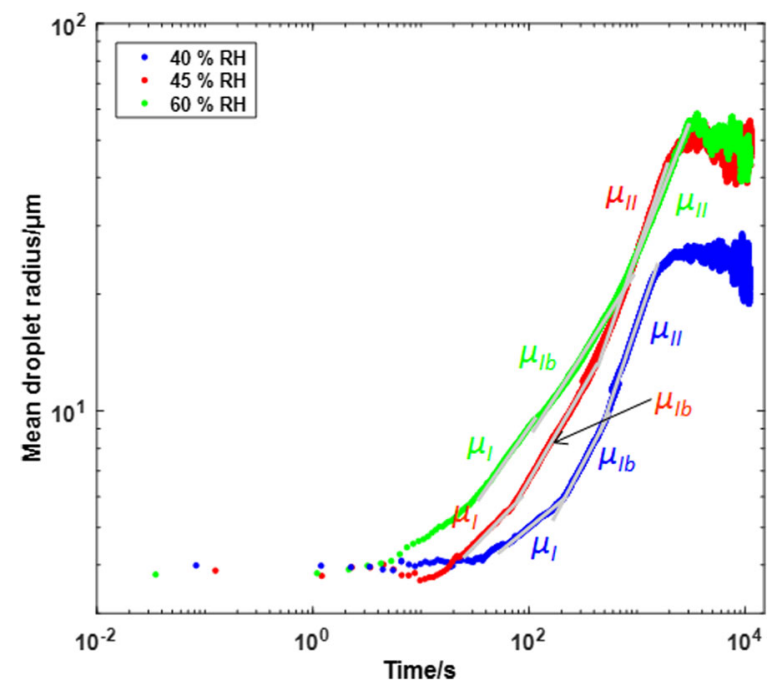

Fig. 8 Mean droplet radius as a function of time for the $40 \%, 45 \%$ and $60 \%$ relative humidity. $0, I, I_{b}$, II and III correspond to the different phases of the curves

limiting the number of coalescence events. Figure 7 shows the decrease of droplet spacing during phase $\mathrm{I}_{\mathrm{b}}$.

The three other phases (0, II and III) are not influenced by the relative humidity. Phase 0 represents the appearance of droplets. The size of the detected droplets depends on the detection sensibility and thus all appearing droplets are the same size (the minimal detected droplet size). However, relative humidity impacts on the droplet appearance duration that influences the growth rate of the previous phases, especially phase $\mathrm{I}_{\mathrm{b}}$. The coalescence events (phases II and III) suppress relative humidity effects on the growth rate, as shown by Castillo et al. [18].

\section{Conclusion}

A custom-made setup was developed to accurately observe the formation of dew on sample surfaces with repeatability. The obtained data were sequences of pictures which were post-processed to detect droplets. The developed program allows the measurement of droplet radius, area, position, etc. Using this equipment, a protocol was built to reproduce literature experiments in order to validate our work. A special effort was made to work with the results of Castillo et al. [18] and to enrich them.

We succeed in reproducing Castillo et al.s [18] experiments thus validating our installation. Moreover, by applying low relative humidity levels and subcooling, we managed to observe droplet appearance and transition phases.

Table $2 \mu$ coefficients for phases $I_{1} I_{b}$ and II of the growth curves

\begin{tabular}{llll}
\hline Relative humidity & $\mu_{l}$ & $\mu_{l b}$ & $\mu_{\|}$ \\
\hline $40 \%$ & $0.23 \pm 0.01$ & $0.53 \pm 0.01$ & $0.81 \pm 0.01$ \\
$45 \%$ & $0.29 \pm 0.01$ & $0.48 \pm 0.01$ & $0.81 \pm 0.01$ \\
$60 \%$ & $0.40 \pm 0.01$ & $0.43 \pm 0.01$ & $0.71 \pm 0.01$ \\
\hline
\end{tabular}


The number of droplets depends on the duration of the appearance step (step 1). This step depends on the time it takes the droplets to reach a permanent size inhibiting the nucleation of new droplets. Thereby, the higher the $\mathrm{RH}$, the lower the number of droplets and, thanks to a better water vapor supply, the higher their non-coalescing growth. Indeed, by decreasing the number of droplets, the increased vapor supply due to $\mathrm{RH}$ is also divided between fewer droplets increasing the amount of vapor provided for each droplet.

The number of droplets has an impact on the intermediate phase between phases without coalescence and phases with established coalescence events. Indeed, during the intermediate phase coalescence starts but is not important enough to obtain $\mu \rightarrow 1$. Thus, the higher the number of droplets, the higher the growth rate of this phase.

This study describing the environmental effect on the condensation dynamics is the first step of research into a new topographical solution reducing the optical impact of the dew on transparent polycarbonate surfaces.

\section{Acknowledgements}

This work was done as part of the OpenLab VAT@Lyon of the Group PSA.

Funding

This work was supported by the Group PSA and the French Ministry of Research.

Competing interests

The authors declare that they have no competing interests.

\section{Publisher's Note}

Springer Nature remains neutral with regard to jurisdictional claims in published maps and institutional affiliations.

\section{Author details}

1 Université de Lyon, Ecole Centrale de Lyon, Laboratoire de Tribologie et Dynamique des Systèmes, UMR 5513, F-69131, 36 Avenue Guy de Collongue, Ecully, France. ${ }^{2}$ PSA Peugeot Citroën, E78943 Vélizy-Villacoublay, France. ${ }^{3}$ Université de Lyon, Ecole Nationale Supérieure des Mines de Saint-Etienne, LGF, UMR 5307, 158 Cours Fauriel, Saint-Etienne, France.

Received: 2 May 2017 Accepted: 29 January 2018

Published online: 17 February 2018

\section{References}

1. Lee A, Moon MW, Lim H, Kim WD, Kim HY (2012) Water harvest via dewing. Langmuir 28(27):10183-10191. https:// doi.org/10.1021/la3013987

2. Macner AM, Daniel S, Steen PH (2014) Condensation on surface energy gradient shifts drop size distribution toward small drops. Langmuir 30:1788-1798

3. Kim JG, Choi HJ, Park KC, Cohen RE, McKinley GH, Barbastathis G (2014) Multifunctional inverted nanocone arrays for non-wetting, self-cleaning transparent surface with high mechanical robustness. Small 10(12):2487-2494. https:// doi.org/10.1002/smll.201303051

4. Park KC, Kim P, Grinthal A, He N, Fox D, Weaver JC, Aizenberg J (2016) Condensation on slippery asymmetric bumps. Nature 531:78-82. https://doi.org/10.1038/nature16956

5. Zamuruyev KO, Bardaweel HK, Carron CJ, Kenyon NJ, Brand O, Delplanque JP, Davis CE (2014) Continuous droplet removal upon dropwise condensation of humid air on a hydrophobic micropatterned surface. Langmuir 30(33):10133-10142. https://doi.org/10.1021/la5004462

6. Briscoe BJ, Galvin KP (1991) The effect of surface fog on the transmittance of light. Solar Energy 46(4):191-197. https://doi.org/10.1016/0038-092X(91)90063-3

7. Zhang L, Li Y, Sun J, Shen J (2008) Mechanically stable antireflection and antifogging coatings fabricated by the layer-by-layer deposition process and postcalcination. Langmuir 24(19):10851-10857. https://doi.org/10.1021/ la801806r

8. Halimeh JC, Roser M (2009) Raindrop detection on car windshields using geometric-photometric environment construction and intensity-based correlation. In: Intelligent vehicles symposium, 2009 IEEE. IEEE. pp 610-615

9. Li Y, Zhang J, Zhu S, Dong H, Jia F, Wang Z, Sun Z, Zhang L, Li Y, Li H, Xu W, Yang B (2009) Biomimetic surfaces for high-performance optics. Adv Mater 21(46):4731-4734. https://doi.org/10.1002/adma.200901335

10. Infante D, Koch KW, Mazumder P, Tian L, Carrilero A, Tulli D, Baker D, Pruneri V (2013) Durable, superhydrophobic, antireflection, and low haze glass surfaces using scalable metal dewetting nanostructuring. Nano Res 6(6):429-440. https://doi.org/10.1007/s12274-013-0320-z

11. Rose JW (2002) Dropwise condensation theory and experiment: a review. Proc Instn Mech Engrs 216:115-128

12. Seo D, Lee C, Nam Y (2014) Influence of geometric patterns of microstructured superhydrophobic surfaces on water-harvesting performance via dewing. Langmuir 30(51):15468-15476. https://doi.org/10.1021/la5041486 
13. Dumont JM (2007) Polycarbonates. Techniques de l'ingénieur, Saint-Denis

14. de Brouwer $\mathrm{H}$, van den Bogerd J, Hoover J (2015) Color stability of polycarbonate for optical applications. Eur Polym J 71:558-566. https://doi.org/10.1016/j.eurpolymj.2015.08.031

15. Sokuler M, Auernhammer GK, Liu CJ, Bonaccurso E, Butt HJ (2010) Dynamics of condensation and evaporation: Effect of inter-drop spacing. EPL (Europhys Lett) 89(3):36004

16. Ucar IO, Erbil HY (2012) Use of diffusion controlled drop evaporation equations for dropwise condensation during dew formation and effect of neighboring droplets. Colloids Surf A Physicochem Eng Asp 411(0):60-68. https://doi. org/10.1016/j.colsurfa.2012.07.002

17. Beysens D (2006) Dew nucleation and growth. C R Phys 7(9-10):1082-1100. https://doi.org/10.1016/j.crhy.2006.10. 020

18. Castillo JE, Weibel JA, Garimella SV (2015) The effect of relative humidity on dropwise condensation dynamics. Int $J$ Heat Mass Transf 80(0):759-766. https://doi.org/10.1016/j.ijheatmasstransfer.2014.09.080

19. Morse BS (2000) Edge Detection. Brigham Young University. Last consultation: 2017/01/30. http://homepages.inf.ed. ac.uk

20. Otsu N (1979) A threshold selection method from gray-level histograms. IEEE Trans Sys Man Cybernet 9:62-66. http://ieeexplore.ieee.org/stamp/stamp.jsp?arnumber=4310076

21. Vera J, Brulez AC, Contraires E, Larochette M, Valette S, Benayoun S (2015) Influence of the polypropylene structure on the replication of nanostructures by injection molding. J Micromech Microeng 25(11):115027

22. Yeh KY, Chen LJ, Chang JY (2008) Contact angle hysteresis on regular pillar-like hydrophobic surfaces. Langmuir 24(1):245-251. https://doi.org/10.1021/la7020337

23. Kashchiev D (2000) Nucleation Basic Theory With Applications. Butterworth Heinemann

24. PALRAM Chemical Resistance of Polycarbonate Products. Last consultation: 2017/01/30. http://www. architectureanddesign.com. http://www.architectureanddesign.com.au/getattachment/cf11179f-d76b-411a-81e7ec8b94139c3d/attachment.aspx

25. Medici MG, Mongruel A, Royon L, Beysens D (2014) Edge effects on water droplet condensation. Phys Rev E 90:062403. https://doi.org/10.1103/PhysRevE.90.062403

26. Guadarrama-Cetina J, Narhe RD, Beysens DA, González-Viñas W (2014) Droplet pattern and condensation gradient around a humidity sink. Phys Rev E 89:012402. https://doi.org/10.1103/PhysRevE.89.012402

27. Beysens D, Steyer A, P. Guenoun P, Fritter D, Knobler CM (1991) How does dew form? Phase Transit 31:219-246

28. Mei M, Yu B, Zou M, Luo L (2011) A numerical study on growth mechanism of dropwise condensation. Int J Heat Mass Transfer 54(9-10):2004-2013. https://doi.org/10.1016/j.ijheatmasstransfer.2011.01.002

29. Beysens D, Knobler CM (1986) Growth of breath figures. Phys Rev Lett 57:1433-1436. https://doi.org/10.1103/ PhysRevLett.57.1433

30. Yu TS, Park J, Lim H, Breuer KS (2012) Fog deposition and accumulation on smooth and textured hydrophobic surfaces. Langmuir 28(35):12771-12778. https://doi.org/10.1021/la301901m

31. Sun X, Damle VG, Uppal A, Linder R, Chandrashekar S, Mohan AR, Rykaczewski K (2015) Inhibition of condensation frosting by arrays of hygroscopic antifreeze drops. Langmuir 31(51):13743-13752

\section{Submit your manuscript to a SpringerOpen ${ }^{\circ}$ journal and benefit from:}

- Convenient online submission

Rigorous peer review

- Open access: articles freely available online

- High visibility within the field

- Retaining the copyright to your article

Submit your next manuscript at $\gg$ springeropen.com 